\title{
Leben und Wesen der Bienen
}




\section{Leben und Wesen der Bienen}

Von

Prof. Dr. H. v. Buttel-Reepen

Mit 60 Abbildungen und einer Tabelle

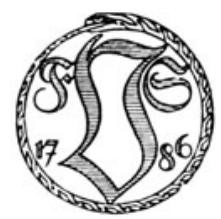

BRAUNSCHWEIG

DRUCK UND VERLAG VON FRIEDR. VIEWEG \& SOHN

1915 
ISBN 978-3-663-03368-4

ISBN 978-3-663-04557-1 (eBook)

DOI $10.1007 / 978-3 \cdot 663-04557 \cdot 1$

Alle Rechte,

namentlich das Recht der Ubersetzung in fremde Sprachen, vorbehalten.

Copyright, 1915, by Friedr. Vieweg \& Sohn,

Braunschweig, Germany.

Softcover reprint of the hardcover 1st edition 1915 


\section{VORWORT.}

Der Text dieser Arbeit lag bereits fertig gedruckt vor, als der Krieg ausbrach. Wann nun die Ausgabe erfolgen kann, ist vorläufig nicht abzusehen. Sollten inzwischen wissenschaftliche Ergebnisse von Bedeutung zur Veröffentlichung gelangen, deren Kenntnisnahme zur Vervollständigung des Inhalts der nachstehenden Darlegungen notwendig oder wünschenswert erscheint, so werden sie in kurzen Hinweisen diesem Vorwort angefügt werden. Im allgemeinen sind wir aber bezüglich der Biologie der Honigbiene zu einem gewissen Abschluß gelangt, wenngleich noch manche Einzelfragen weiterer Erledigung harren. Der starke Fluß der Geschehnisse auf dem hier in Betracht kommenden Gebiet, wie er sich in den letzten 10 bis 15 Jahren zeigte und der die Herausgabe dieses Buches zum Teil verzögerte, löst sich jetzt in einzelne kleinere Verzweigungen auf, die sich in noch unerforschte Gebiete verlieren, in denen wohl noch Ergänzungen und Bestätigungen, aber keine grundlegenden Veränderungen zu erwarten sind.

Es dürfte wohl richtig sein, daß in manchen Kreisen bis in die jüngste Zeit hinein eine gewisse Unsicherheit bezüglich der allgemeinen Biologie der Honigbiene herrschte. Der Naturwissenschaftler sah sich, von Einzelfragen abgesehen, in der Hauptsache auf die Imkerliteratur verwiesen, die aber im wesentlichen nur die reine Praxis behandelt und dort, wo sie über dieses Gebiet hinausgelangt, recht Unsicheres bietet, zumal sie ohne Quellennachweise zu arbeiten pflegt.

$\mathrm{Ob}$ die nachstehenden Darlegungen hier nunmehr, neben der Zanderschen eine völlig andere Ausgestaltung zeigenden Arbeit (1913), eine genügende Grundlage gewähren, wage ich nicht zu entscheiden, da mir natürlich am besten bewußt ist, wieviel zu wünschen übrig bleibt, zumal meine Ausführungen ursprünglich für die im gleichen Verlage erscheinende Serie wissenschaftlicher Monographien "Die Wissenschaft" bestimmt waren, die eine starke Begrenzung des Raumes vorschreibt und demnach nicht eine alle Einzelheiten 
erschöpfende Biologie und Psychobiologie gegeben werden konnte. Nichtsdestoweniger findet sich alles Wichtige herangezogen. Als die Fülle des Stoffes diese Begrenzung sprengte, war die Ausführung schon zum Teil festgelegt, so daß wesentlich erscheinende Angliederungen und Ergänzungen ihre besondere Erledigung verlangten. Ein möglichst sorgfältiges alphabetisches Register und zahlreiche Seitenverweise werden aber trotzdem eine leichte Orientierung ermöglichen.

Die anfängliche Absicht, jedem Kapitel ein Literaturverzeichnis beizugeben, mußte wegen der Fülle der Literatur (zitiert wurden etwa 550 Arbeiten), die wegen der notwendigen Wiederholungen eine zu große Raumbelastung darstellten, aufgegeben werden.

In Rücksicht auf die Dozenten, besonders auch an landwirtschaftlichen Hochschulen usw., ist das Kapitel über Wohnungen und Wabenbau etwas ausführlicher gestaltet, doch ich bemerke ausdrücklich, daß ich mich keineswegs mit dem Bienenwirtschaftlichen befasse. Hierfür existieren zahlreiche Lehrbücher der Bienenzucht, zu denen dieses Werk die notwendige Ergänzung und Führung bieten will. Der Naturfreund wie auch der Imker dürften ihr Interesse für das Leben der Bienen sich vertiefen sehen, wenn sie dem eigentlichen Wesen dieses so wunderbaren Familienstaates nähertreten und in Gebiete und biologische Verhältnisse hineinschauen, die der eigentlichen Praxis ferner liegen, die zu kennen aber vielfach auch für letztere von Wert sind nach dem Spruche v. Berlepschs: „Lernt Theorie, sonst bleibt ihr Stümper in der Praxis euer Leben lang".

Alle eingeklammerten Jahreszahlen beziehen sich auf das Literaturverzeichnis.

Den Herren Verlegern bin ich für vielfaches Entgegenkommen zu größtem Dank verpflichtet.

Oldenburg i. Gr., 31. August 1914.

Buttel-Reepen. 


\section{Inhaltsverzeichnis.}

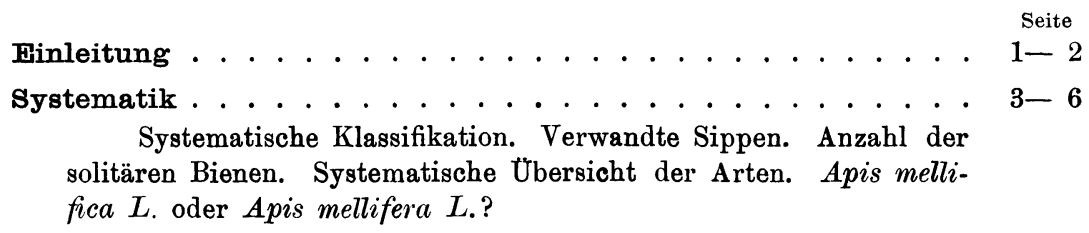

\section{Das Leben der Bienen.}

Erstes Kapitel.

Die Urheimat der Bienen.

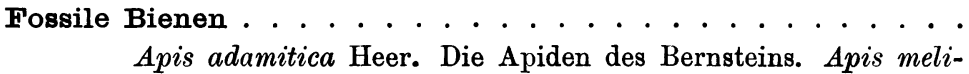
ponoides Butt.-Reep. Electrapis meliponoides Butt.-Reep. Apis (?) dormitans Heyden. Apis henshawi Cockerell.

Die Urheimat . . . . . . . . . . . . . . . . . . . . . . . . . 14-18

Wo konnten zuerst blumensuchende Insekten entstehen? Wann begann die Staatenbildung? Die Ausbreitung in vorgeschichtlicher Zeit. Einfluß der Eiszeit.

Zweites Kapitel.

Die geograph. Verbreitung der Honigbiene (Apis mellifica $\mathrm{L}$ ), ihrer Varietäten und der übrigen Apis-Arten.

Europa. Die dunkle deutsche Biene . . . . . . . . . . 20-21

Exporte nach Amerika, nach Australien, nach Asien.

Die italienische Biene . . . . . . . . . . . . . . . . . 22-23

Importe nach Deutschland. Exporte nach Amerika, nach Asien, nach Australien.

Die cyprische Biene . . . . . . . . . . . . . . . . . . 23

Einführung nach verschiedenen Ländern.

Die syrische (palästinische) Biene . . . . . . . . . . . . . . . 23-24

Verbreitung. 
Die kaukasische Biene. . . . . . . . . . . . . . 24

Die Krainer oder Norische Biene . . . . . . . . . . . . . . . . 24

Die griechische Biene . . . . . . . . . . . . . . . . . . . . . . 24-25

Afrika. Die ägyptische Biene . . . . . . . . . . . . . . . . . 25

Die gelbe afrikanische Biene . . . . . . . . . . . . . . . . . . 25

Die dunklen afrikanischen Bienen . . . . . . . . . . . . . . 26

Die schwarze Madagaskarbiene und Varietäten.

Verbreitung nach und von Afrika . . . . . . . . . . . . . . . 26

Die kleinasiatisch-griechischen Varietäten . . . . . . . . . . . 26

Weitere europäische Bastardbienen . . . . . . . . . . 26-27

A sien. Die indische Biene. . . . . . . . . . . . . . . . . . . 27

Die chinesische Biene.

Die indische Riesenbiene . . . . . . . . . . . . . . . . . . . . 27

Die indische Zwergbiene. . . . . . . . . . . . . . . . . 28

\section{Drittes Kapitel.}

Polymorphismus und Morphologisches.

Polymorphismus . . . . . . . . . . . . . . . . . . . . . 29-30

Typische und atypische Formen. Normale Zusammensetzung des Volkes.

Morphologisches . . . . . . . . . . . . . . . . . . . 30-34

Viertes Kapitel.

Die Parthenogenese bei der Honigbiene.

Geschichte und Wesen der Parthenogenesis . . . . . . . . . . 35-45

Die Zahl der Spermien im Receptaculum seminis.

\section{Fünftes Kapitel.}

I.

Die stammesgeschichtliche Entstehung des Bienenstaates. Der Einfamilienstaat.

Die Abstammung von den Grabwespen. . . . . . . . . . . . . 45-46

Aus dem Leben der solitären Bienen . . . . . . . . . . . . . . 46-60

Soziale Instinkte bei den Solitären. Gemeinsame Uberwinterung. Gemeinsame Nachtruhe. Gemeinsamer Flugkanal. Der Kontakt von "Mutter" und „Kind“. Anfang der Staatenbildung. Fortschritt zur ersten Kolonie.

Die Hummeln - ein wichtiges Übergangsglied . . . . . . . . 61-62 tären.

Brutpflege bei den Hummeln. Andauernde Fütterung bei Soli-

Die Ausbildung typischer Arbeiter . . . . . . . . . . . . . . . 62-64

Der Fortschritt im Zellenbau.

Biologischer Stammbaum . . . . . . . . . . . . . . 65 
II.

Zur Geschichtsphilosophie des Bienenstaates. Seite

Polygyner oder monogyner Ursprung der Staatenbildung . . 66-90

Zweierlei Arten von Polygynie. Die Sippenpolygynie. Anor-

male Polygynie bei Sozialen. Die Polygynie einiger neotropischer

Sozialen.

Das Wesen der verschiedenen Schwarmarten . . . . . . 75-78

Die neotropischen Wespenschwärme.

Der Wert tropischer Formen für die Stammesgeschichte . . 78-88

Ameisenarbeiter erzeugen Arbeiter. Die neotropischen

Hummelarbeiter. Die sozialen paläarktischen Hymenopteren-

formen primitiver als die neotropischen.

Weiteres über den Schwärminstinkt . . . . . . . . . . . 85

Ist die Monogynie eine Folge des Klimas? . . . . . . . . . . 86

Präformation oder Fpigenese?. . . . . . . . . . . . . . 87 Königin.

Vererbung durch die Königin. Die Instinktsverluste der

Ist die Monogynie bei Apis ein Ausleseprozeß ? . . . . . . . 89-90

Sechstes Kapitel.

Wabenban und Wohnungen der Honigbiene.

Stabilbau und Mobilbau. . . . . . . . . . . . . . . 91-109

Die Erfindung der beweglichen Wabe.

Siebentes Kapitel.

Biologie.

Biologisches . . . . . . . . . . . . . . . . . . . . . . . 109-139

Eiablage im Winter 109. Anzahl der Zellen 110. Gewicht und Anzahl der Bienen 110. Honigvorrat 110. Die Anordnung der Zellen 110. Eiablage im Frühling 111. Die Stellung der Königin im Staat 111. Die Entwicklungsstadien der Brut 113. Die Zusammensetzung des Futterbreies 113. Brutammen 114. Das Vorspiel und der Orientierungssinn 114. Ist der Futterbrei ein Drüsensekret? 115. Wasserbedarf zur Futterbreierzeugung und im Stocke 115. Die Drohnenzellen 116. Der Bau der Weiselzellen 116. Das Auftreten der Schwärmstimmung 116. Die Weiselnäpfchen 117. Die Zahl der Weiselzellen 117. Einspinnen der Larven und die sogenannte "Nymphenhaut" 117. Die Zusammensetzung der Brutdeckel 118. Das Engerwerden der Zellen durch die Kokongespinste 118. Temperatur im Volke 119. Vorbereitungen zum Schwärmen 119. Der Schwarmakt 120. Die Spurbienen 122. "Ausreißen" der Schwärme 123. Der Vorschwarm 124. Die Nachschwärme 124. Das Tüten und Quahken der jungen Königinnen 124. Das Ausziehen des Nachschwarmes 125. Die Neugründung einer Kolonie durch einen Nachschwarm 126. Hochzeitsvorspiel 126. Die Verhängung 127. Mißverständnisse im 
Volk 127. Eine Begattung während des Schwärmens 128. Begattung einer Königin von Vespa rufa 129. Die Drohnenkugeln beim Befruchtungsausflug der Bienenkönigin 129. Arbeitsleistung des Nachschwarmes 130. Leistung der Königin bei der Eiablage 130. Zahl der Eier. Der „Hofstaat" der Königin 131. Lebensdauer der Biene 132. Lebensdauer der Königin 133. Beobachtungen am Wagvolk 134. Was ist Nektar und was ist Honig? 135. Honigverbrauch des Volkes 137. Honigerträge eines Volkes 138. Tagesleistung im Honigertrag 138. Die Drohnenschlacht 138. Einwinterung 139.

Achtes Kapitel.

Das Rätsel des Pollensammelns.

Ausführung und Geschichte. . . . . . . . . . . . . . . . . 140-149

Beschreibung der Organe. Die sogenannte Wachszange.

Neuntes Kapitel.

Die Vorgänge bei der Wachsabscheidung.

Geschichtliches und Ausführungsart . . . . . . . . . . . . . 150-158

Wo wird das Wachs ausgeschieden? Abnorme Wachsausscheidung bei der Honigbiene. Wie gewinnt die Biene die Wachslamellen zur Verarbeitung?

\title{
Vom Wesen der Honigbiene.
}

\author{
Zehntes Kapitel. \\ Die Sinne der Biene.
}

I.

Der Ortssinn, Orientierungssinn, die „Heimkehrfähigkeit“, der kinästhetische „Winkelsinn", Raumsinn usw. . . . . 159-164

Was ist das Ortsgedächtnis? Weite des Flugkreises. Der Orientierungssinn der Königin und der Drohnen. Das Betäuben der Bienen. Der Schwarmdusel. Verharren alter Eindrücke.

II.

Farben- und Formensinn . . . . . . . . . . . . . . . 164-171

Können die Bienen die Farben als solche unterscheiden? Besonderes Orientierungsvermögen. Der Lichtsinn der Bienen. Sind Schwarmbienen besonders positiv heliotropisch? Was zieht die Bienen zu den Blumen? Assoziation von Eindrücken.

III.

Das Sehvermögen der Bienen . . . . . . . . . . . . . 171-178

Die Bedeutung der Stirnaugen und der Facettenaugen. Die Beweglichkeit der Augen bei der Biene. Das Sehen im Dämmern vermittelst der Stirnaugen. Sehschärfe. Sehweite. 
IV.

Das angeblich schlechte Geruchsvermögen der Bienen. Irritabilität der Bienen. Haben die Wespen einen besseren Geruchssinn als die Bienen? Die vitale Bedeutung des Geruchssinnes. Das Verfliegen der Bienen. Der Königingeruch. Die Ergatogynen im Bienenstaat. Weisellosigkeit und Geruchsvermögen. Wie merken die Bienen den Verlust der Königin? Das Anhaftende des Arbeiterinnengeruches. Die Befunde McIndoos über neue Geruchsorgane.

V.

Das Mitteilungsvermögen der Bienen und ihr Gehörssinn .

$190-209$

Lautsprache. Sterzelton. Nachahmungstrieb. Antennensprache. Fluglochwache. Können die Bienen hören? Gehörvermögen der Ameisen. Die Forelschen Flaschen als Gehörsorgane. Die Membranplatten als Gehörsorgane. Neuerdings entdeckte chordotonale Sinnesorgane der Biene. Hörvermögen der Hummeln und Wespen. Ein seltsames Phänomen.

Elftes Kapitel.

\section{Die Instinkte der Biene.}

Die Instinkte im Lichte deszendenztheoretischer Betrachtung

Atavistische Erscheinungen im Bienenstaat 210. Atavistische Merkmale der Königin 210. Klammerorgane der Drohne 211. Die atavistische Bauart der Weiselzelle 211. Die Größendifferenz zwischen Königin und Arbeitern 213. Die Sporen (Calcaria) bei der Honigbiene 215. Atavistische Instinkte beim Eintreten der Weisellosigkeit 217. Drohnenzellenbau nach Königinverlust 220. Drohnen von Afterköniginnen vollwertige Männchen 221. Warum werden die Drohnen im weisellosen Volk nicht abgestochen? 222. Das Verkitten zum Teil eine atavistische Reminiszenz 222. Atavistisches beim Schwärmen 223. Instinktsirrungen 224. Arbeiter in Buckelbrut 226. Taube Eier 226. Das Einballen der Königin 227. Anfallen der eigenen Nestgenossen 228. Sonstige Instinktsirrungen 229.

Besondere Instinkte . . . . . . . . . . . . . . . . . . . . . 230-244

Der Zellenbauinstinkt 230. Der Spieltrieb 234. Der Zeitsinn 234. Der Temperatursinn 234. Wärme im Hornissennest 236. Der Reinlichkeitsinstinkt 236. Warum kehren die Bienen halbbeladen heim? 236. Zahl der Ausflüge 236. Kennen die Bienen den Bienenvater? 238. Neu nach Volksbildung hinzugewonnene Instinkte 239. Fälschlich zugeschriebene Instinkte 239. Die Drucktheorie zur Geschlechtsbestimmung bei der Eiablage 240. Die Biene als Dysteleologe 241. Das Modifikationsvermögen der Instinkte 241. Dauerreize verlaufen in völlig veränderten Bahnen 242. 
Zwölftes Kapitel.

Zur Psychologie der Bienen.

\section{Anmerkungen.}

Anmerkung 1. Geologische Tabelle nebst Angabe der Versteinerungen . . . . . . . . . . . Hinter S. 300 eingefügt Anmerkung 2. Beobachtungen an Eucera longicornis . . . . 253-254 Anmerkung 3. Die Staatenbildung im Sinne Wlad. Wagners 254-256 Anmerkung 4. Der Richtungssinn Gaston Bonniers. Der Richtungssinn W lad. Wagners. Der Richtungssinn beim Menschen. . . . . . . . . . . . . . . . . 256-259

Literatur . . . . . . . . . . . . . . . . . . . . . . . . 260-282

Autorenregister . . . . . . . . . . . . . . . . . . . . 283-286

Alphabetisches Sachregister. . . . . . . . . . . . . . . . . 287-300

Geologische Tabelle . . . . . . . . . . . . . . . 301 


\section{Verzeichnis der Abbildungen.}

1 Apis adamitica Heer . . . . . . . . . . . . . . . . 9

2-5 Umrisse der Hinterbeine III von Apis mellifica 9 , ㅇ; Apis meliponoides, Melipona anthidioides $9 . . . . . . . . . .11$

6-8 Königin, Drohne und Arbeiterin. . . . . . . . . . . . . . . 30

9 Embryo im Bienenei im Alter von etwa $2^{1 / 2}$ Tagen . . . . . . . 31

10 Durchschnitt durch eine erwachsene Arbeitsbiene . . . . . . . . 33

11 Geschlechtsapparat der Königin . . . . . . . . . . . . . . . 37

12 Befruchtungsvorgänge im Bienenei etwa 15 Minuten nach Ablage des Eies . . . . . . . . . . . . . . . 39

13 Nest von Osmia papaveris . . . . . . . . . . . . . . . 47

14 Anormales Nest von Osmia papaveris . .. . . . . . . . . . . . 47

15 Nest einer Osmia in einem Brombeerstengel . . . . . . . . . . . 49

16 Lehmwabe von Halictus quadricinctus . . . . . . . . . . . . . . 56

17 Lüneburger Stülpkorb mit vorlagernden Bienen . . . . . . . . . 91

18 Blick in einen umgedrehten Bienenkorb . . . . . . . . . . . . . 92

19 Heidebienenstand . . . . . . . . . . . . . . . . . . 93

20 Kastenbienenzucht . . . . . . . . . . . . . . . . . . . . 94

21 Ausgebautes Rähmchen mit allen Zellenarten. . . . . . . . . . . 95

22 Rahmenbude Francois Hubers . . . . . . . . . . . . 97

23 Bienenstock von P. J. Prokopovitsch . . . . . . . . . . 98

24 Rähmchen von Szarka................. 99

25-27 Stäbchen nach Dzierzon. . . . . . . . . . . . . . . . . . 99

28 Berlepschrähmchen . . . . . . . . . . . . . . . 100

29 Ältestes Rähmchen vom Jahre 1683 . . . . . . . . . . . . . . . 103

30 Christsches Magazinkästchen. . . . . . . . . . . . . . 104

31 Griechischer Bienenkorb . . . . . . . . . . . . . . . 104

32 Mobilbeute Della Roccas vom Jahre 1790 . . . . . . . . . . 105

33 Ägyptischer Bienenstand . . . . . . . . . . . . . . . . 107

34 Querschnitt durch eine Wabe und die prismatischen Zellenböden . 111

35 Eier auf dem Grunde der Zellen und stark vergrößertes Ei . . . . 111

36 Ein Volk im Strohkorb unmittelbar vor dem Ausschwärmen . . . 120

37 Ein sich anlegender Schwarm . . . . . . . . . . . . . . . . . . 122

38 Königin und Drohne in Verhängung . . . . . . . . . . . . . . . 128

39 Wagvolktabelle................... 134

40 Biene mit Pollenhöschen . . . . . . . . . . . . . . . . . . 140 
Fig. $\quad$ Seite

41 Innenseite des linken Hinterbeines der Arbeitsbiene . . . . . . . . . 141

42 Außenseite des linken Hinterbeines der Arbeitsbiene. . . . . . . . . 143

43 Außenseite der Tibia des linken Hinterbeines. Körbchenglied . . . . 144

44 Biene, die Pollenballen durch das mittlere Beinpaar festdrückend . . . 145

45 Biene, mit dem Pollenkamm Blütenstaub kämmend . . . . . . . . . 146

46 Anwachsen der Pollenballen durch die Schubmethode . . . . . . . . 147

47 Falsche Darstellung der Wachsausscheidung an den Bauchsegmenten . 153

48 Richtige Darstellung der Wachsausscheidung . . . . . . . . . . . 153

49 Abnorm starke Wachsausscheidung . . . . . . . . . . . . . . . 154

50 Wie das Wachsblättchen erfaßt wird . . . . . . . . . . . . 157

51 Bauchansicht einer Arbeiterin, die im Begriff ist, ein Wachsblättchen zu entfernen . . . . . . . . . . . . . . . . . 157

52 Seitenansicht einer Arbeiterin, die ein Wachsblättchen zum Munde führt . . . . . . . . . . . . . . . . . . 157

53 Rückenansicht einer Arbeiterin mit den McIndooschen Geruchsporen 188

54 Durchschnitte durch stark vergrößerte Geruchsporen nach McIndoo 189

55 Die Sinnesorgane auf den Fühlern von Apis mellifica . . . . . . . . 200

56 Hörorgan in den Beinen der Apis mellifica . . . . . . . . . . . . . 204

57 Weiselzellen auf künstlicher Mittelwand. . . . . . . . . . . . . . . 212

58 Der Sporn (Calcar) bei Apis mellifica. . . . . . . . . . . . . . . 216

59 Die Sporen-Rudimente an den Hinterschienen der Puppen der Arbeiterin,

Drohne, Königin und von Bombus distinguendus . . . . . . . . . 217

60 Das Abtrommeln eines Volkes . . . . . . . . . . . . . . . . . 243 